\title{
Salivary gland endoscopy in children: A systematic review
}

\author{
Leonardo Silva ${ }^{1 *}$, Gean Babicsak ${ }^{1}$, Ricardo Landini Dolci ${ }^{1}$ \\ 1Department of Otorhinolaryngology, Faculdade de Ciências Médicas da Santa Casa de São Paulo, São Paulo, SP, Brazil
}

Study conducted at the Department of Otorhinolaryngology of Faculdade de Ciências Médicas da Santa Casa de São Paulo, São Paulo, SP, Brazil

Article received: 9/8/2015 Accepted for publication: 10/19/2015

*Correspondence: Departamento de Otorrinolaringologia Address: Rua Martiniano de Carvalho 864, cj. 1001 São Paulo, SP - Brazil Postal code: 01321-000 leosilva@uol.com.br

\section{SUMMARY}

Objective: To review studies on sialendoscopy (SE) of the salivary glands in children focusing mainly on the indications, endoscopic findings, and effectiveness of the procedure.

Method: The electronic databases searched were Pubmed, Scielo, and Cochrane. The search was conducted by two researchers independently, following inclusion and exclusion criteria. A third author analyzed sources of conflict. In the first stage they were discarded by reading the articles title that had no relation to the purpose of the study and then evaluated the abstracts of each study. In these two initial phases 37 articles were excluded. Articles not excluded by the selection criteria have been retrieved and assessed in full. Seven articles had their data extracted and were compared.

Results: The literature search parameters listed allowed the recovery of 44 articles. After applying the exclusion criteria, seven studies were included in this review representing 207 patients undergoing with ages ranging from 1 to 16 years. All studies except one underwent SE under general anesthesia. The juvenile recurrent parotitis was the main clinical diagnosis related with SE procedures $(\mathrm{N}=152)$. The number of inflammatory attacks per patient per year was the parameter for indication of SE. The efficacy of the procedure was considered high by all authors ranging between 83 and $93 \%$ in larger series evaluated.

Conclusion: Sialendoscopy is a safe and effective procedure for the diagnosis and treatment of recurrent inflammatory diseases of salivary glands in children.

Keywords: child, endoscopy/methods, salivary gland diseases.

\section{INTRODUCTION}

Recurrent inflammatory diseases of the major salivary glands that occur in childhood and adolescence, for the most part, do not have a defined etiology. Usually, when there is simultaneous involvement of two or more glands, etiopathogenic hypotheses end up being related to autoimmune or genetic factors. ${ }^{1}$ However, when repeated inflammation affects only one of the glands, obstructive factors such as stenosis, malformation, and ductal obstruction by calculus and/or debris are a more likely diagnostic hypotheses. ${ }^{2}$

Given the difficulty of the etiologic diagnosis, despite the different methods available, the treatment ends up being in large part directed to symptomatic relief. ${ }^{1}$

Sialendoscopy (SE) is a relatively new method for diagnostic and therapeutic approach to salivary gland disorders in children, initially proposed in $1997 .{ }^{3}$
Various infections of major salivary glands, both in adults and children have been diagnosed and treated by SE. ${ }^{2}$ Using an endoscope inserted through the papilla of the affected gland, a direct view of the ductal system is obtained. Although the main objective of SE is to evaluate the anatomic integrity of the salivary ducts, it is possible to act therapeutically in certain cases during the examination procedure preserving the gland and restoring its function. ${ }^{1,2} \mathrm{SE}$ is a procedure considered minimally invasive, safe, and shows low complication rates. It is indicated as an alternative to open surgery. ${ }^{4}$

Even though the technique has been proposed years ago, its indication for the treatment of children is recent. The development of more sensitive instruments has enabled the transfer of skills and experience obtained in procedures performed in adults to pediatric patients. ${ }^{2,4}$ 
Given that SE in children is a more recent technique than it is in adults, the literature on the subject is still relatively poor. ${ }^{1}$ Many cases of salivary gland disorders in children are reported in series encompassing different ages and conditions. Few studies in the literature report salivary gland disorders in series exclusively directed to conditions affecting the pediatric population. ${ }^{5}$

This fact, coupled with the lack of studies published in the Brazilian literature on SE in the pediatric population, motivated this study. Our objective was to review the studies related to SE in children focusing on indications, endoscopic findings, and the effectiveness of the procedure.

\section{Method}

\section{Literature search strategy}

This study was registered at the PROSPERO platform under number CRD42015019590. The literature search was structured using the PICO strategy and aimed to retrieve publications on sialendoscopy in children. Keywords included "child," "endoscopy/methods," and "salivary gland diseases." We did not use free terms. The Boolean operator employed was "AND" using the combination: Child AND Endoscopy/methods AND Salivary Gland Diseases.

The search was conducted by two researchers independently, following the inclusion and exclusion criteria. A third author was responsible for the analysis of potential sources of conflict.

Electronic databases used in this research were Pubmed, Scielo and Cochrane.

\section{Article selection}

Inclusion criteria:

I. articles that reported the experience of SE exclusively in populations aged between 0 and 16 years old;

II. original articles with clear description of the sample and methods;

III. description of the technique used for SE;

IV. objective presentation of the indication for the procedure;

V. description of the endoscopic findings;

VI. presentation of treatment efficacy.

\section{Exclusion criteria:}

I. articles that reported the experience of SE in populations outside the age range between 0 and 16 years old;

II. articles written in languages other than English, Portuguese, Spanish or Italian;

III. study design - case reports, literature reviews, editorials; IV. animal studies.
There was no limitation regarding the date of publication of the studies.

\section{Data analysis and extraction}

After recovering the items according to the rules described above, the following steps were followed sequentially:

1. Analysis of titles. Titles that were not relevant to the topic proposed lead to exclusion from the study.

2. Analysis of summaries. We evaluated all the summaries of articles selected based on characteristics of the title.

3. Access and analysis of full articles. Articles that met the inclusion criteria based on their summaries were accessed and analyzed in full.

4. Organization and protocol completion. The articles accessed in phase 3 had their data of interest selected and included in a previously organized spreadsheet in order to meet the objectives of this article. The data extraction protocol was organized in Excel (Microsoft Office $2010 \AA)$. In each of the articles included in the evaluation, the following variables were analyzed: sample size, the study objective, method, results, sample characterization (gender, age), mean age of onset of symptoms, patient age in the indication of sialendoscopy, number of episodes of inflammation indication criteria of the procedure, type of anesthesia used, the number of recurrences after surgery, radiological diagnosis, endoscopic diagnosis, and complications.

Possible sources of conflict and disagreement in each stage were resolved by a third investigator. No contact was made with the authors of the primary studies, since there was no doubt in completing the protocols.

\section{Results}

The search in the databases following the above described search strategy allowed the identification of 44 articles in the following proportion for each database: PubMed (43), The Cochrane Library (1), Cochrane Plus Library (0) Resumos de Revisões Sistemáticas em Português (0), Scielo (0) (Figure 1).

The 44 articles retrieved were submitted to an initial assessment by two independent researchers that excluded 37 articles based on title and summary, with regard to the inclusion and exclusion criteria described above. The seven remaining works were accessed and incorporated into the study. To ensure that the criteria for data selection and extraction could be applied consistently by more than one researcher, three randomized studies were previously tested in a pilot. 


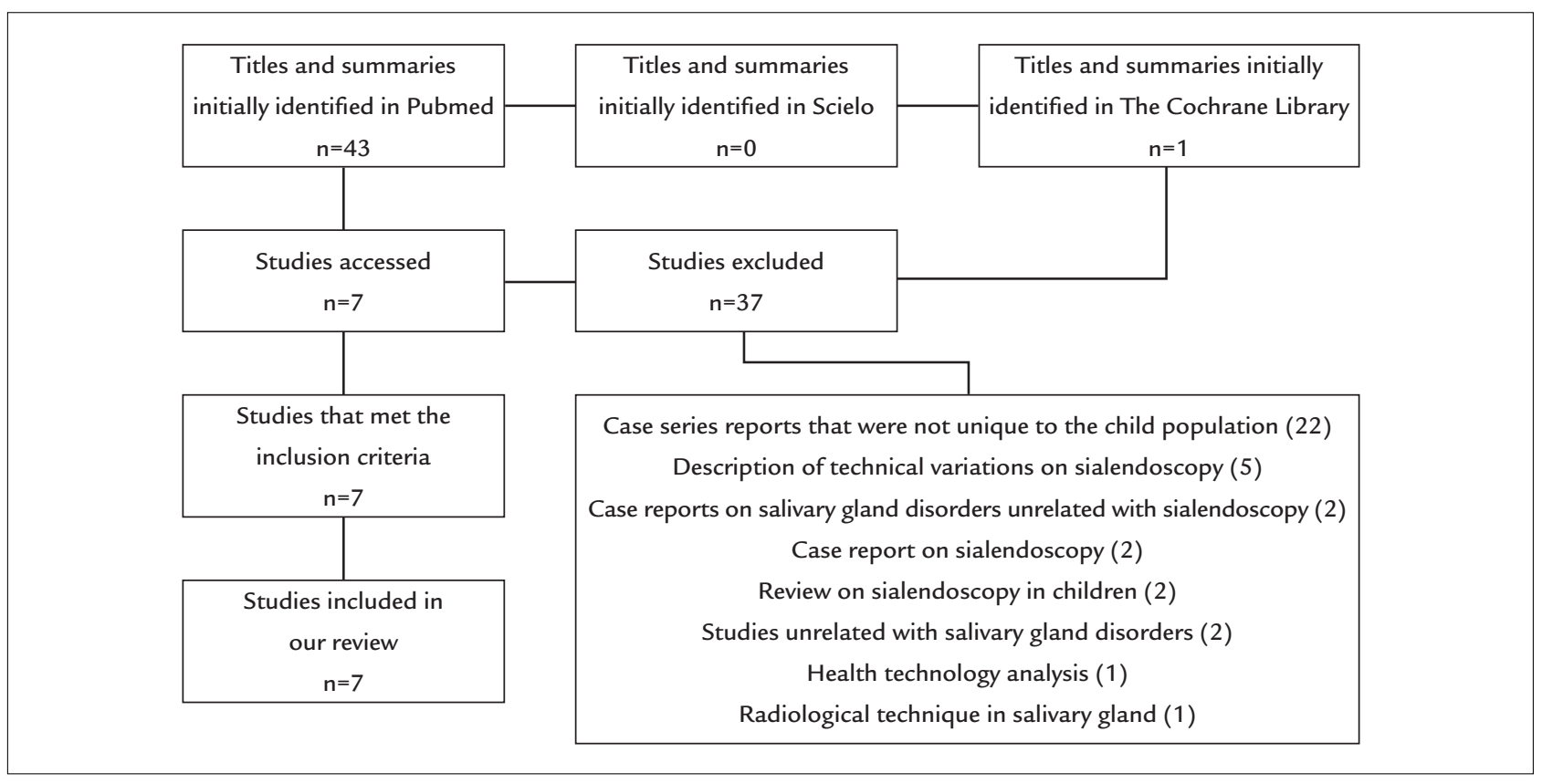

FIGURE 1 Diagram of the study selection process. Although many studies were excluded by multiple reasons, the following order was adopted for the exclusion criteria: articles that did not solely report the experience in populations between 0 and 16 years of age; case reports; literature review; articles written in languages other than English, Portuguese, Spanish or Italian; articles unrelated to the purpose of this study; editorials; and animal studies.

There were no sources of conflict and the third evaluator endorsed the decisions made by the two other researchers. The heterogeneity of the studies given mainly by differences in the characteristics of the populations studied, the SE indication criteria, and follow-up after the procedures precluded a meta-analysis. Despite the limited sample, clinically relevant results could be extracted from this review.

The set of seven studies has an $\mathrm{N}$ of 207 patients with ages ranging from 1 to 16 years undergoing SE. All authors, except one, conducted the SE under general anesthesia (198 patients under general anesthesia and nine under local anesthesia). Infantile recurrent parotitis (IRP) was the clinical diagnosis that led to the indication of SE in more patients, totaling 152 procedures.

The frequency of glandular inflammation attacks was the criterion used by all authors to perform SE, with two sialadenitis in 1 year being the minimum criteria found in this review. ${ }^{6}$

Endoscopic findings of IRP were described by all of the authors as paleness of the lining of the ducts, and shortage of blood vessels which typically run alongside this layer. Other endoscopic findings were ductal stenoses (single and multiple), mucous plugs, debris accumulation, and dilations. ${ }^{7-9}$ As for remission rate recurrent inflammation after the procedure, the rates found by Ardeklan et al. ${ }^{4}$ were $86 \%$ at 6 months of follow-up, including 50 patients; Capaccio et al. ${ }^{7}$ found $65 \%$ of remission after 30 months, with 14 patients treated with SE; and Shacham et al. ${ }^{6}$ showed a $93 \%$ remission rate, with a follow-up of 6 to 36 months after treatment, and 65 patients treated surgically. The authors show consensus to conclude that $\mathrm{SE}$ is an effective and safe technique in the diagnosis and treatment of IRP and that SE probably acts on the anatomy and physiology of the duct. As a result of the passing of the device and the irrigation and flushing of the ducts, many of the abnormalities related to the IRP, such as stenoses, debris, and mucus plugs, are eventually resolved by improving the drainage as well as the prognosis and viability of the gland in the medium and long term. ${ }^{4,-8}$

In the studies accessed for purposes of this review, 22 cases of children with sialolithiasis (SL) were described. Of these, 20 patients underwent ultrasound investigation (USG) prior to SE. Radiological diagnosis of SL was established in 11 of 20 cases $(55 \%)^{2,8,9}$

Among the concurrent endoscopic findings, presence of calculi, mucous plugs, and ductal stenoses were the most frequent. ${ }^{2,9}$ The effectiveness of SE for the diagnosis of SL was $100 \%$. However, there was great variation in the effectiveness of the endoscopic treatment of SL, which was $100 \%$ of resolution at 18 months of follow-up $(\mathrm{N}=12)$ and $50 \%$ at 23 months $(\mathrm{N}=4){ }^{8}$ 


\section{Discussion}

The small diameter of the ductal system of the salivary glands in pediatric patients makes SE a more complex procedure than it is in adults. ${ }^{6}$ The direct view of the ducts by SE allows the diagnosis of diseases such as ductal stenoses and mucous plugs with greater accuracy compared to other diagnostic methods currently available. ${ }^{10}$ In addition to being a diagnostic method, SE enables flushing of the glandular ducts, removal of debris, ductal dilatation, and direct instillation of corticosteroids or antibiotics. ${ }^{8}$ Thus, compared to other methods of treatment, it has the advantage of breaking the glandular inflammatory cycle.

Among the indications for SE in the pediatric population, IRP is the most common; IRP is the second cause of increase in the volume of the parotid gland in children, the first cause being mumps. The first peak of incidence of IRP occurs between 3 and 6 years of age and the second peak, between 9 and 10.5 The diagnosis of IRP is based on clinical criteria and can be complemented by ultrasound and sialography. ${ }^{2,7}$ Although considered a benign and often self-limiting disease, some authors have observed that some of the patients diagnosed with IRP maintained inflammatory conditions into adulthood. In these cases, the long period of recurrent inflammation since childhood can cause irreversible glandular abnormalities. ${ }^{6}$ The endoscopic findings most commonly described in IRP include dilated glandular papillae, paleness with decreased vascularization of the ductal system, and stenoses with the presence of debris. SE should be considered as an option for diagnosis and treatment of IRP. ${ }^{6,9}$ Different authors support the efficacy of the method as a treatment for IRP. In a series of 70 children treated for IRP, the authors found that in $93 \%$ of patients, a single procedure was sufficient for the resolution of symptoms. ${ }^{6}$ In another study, there was resolution of symptoms in $83 \%$ of 23 patients undergoing a single procedure. Konstantinidis et al. studied seven patients, of which only one required an extra procedure, and two others had one episode of recurrence after 1 year (83\% improvement with one procedure). ${ }^{5}$ In a study by Jabbour et al., five patients were treated, of which three children had 1 or 2 episodes of recurrence, but the frequency of symptoms was uniformly decreased. ${ }^{1}$ Differential diagnoses of IRP include infections, Sjögren's syndrome and SL. ${ }^{6}$

Although SL is infrequent and represents less than 5\% of all cases reported in the literature, its occurrence in childhood is probably underestimated in view of the sensitivity limitation of diagnostic tests. ${ }^{9}$ Calculi sized up to $2 \mathrm{~mm}$ are considered as the lower limit for detection of different radiological methods (ultrasound, computed tomography, and magnetic resonance imaging). ${ }^{11}$ Thus, the radiological diagnosis of salivary gland calculus in the pediatric population is less sensitive than in adults. ${ }^{2}$

In children with recurrent swelling of the salivary gland, especially when the involvement is unilateral, the presence of calculi should be one of the diagnostic hypotheses even when the radiological examination is considered normal. Compared with other radiological methods, SE is not just a diagnostic method but a possibility of treating the patient by removing calculi. ${ }^{1,2}$ Thus, SE has been considered a safe technique for diagnosis and treatment of sialolithiasis in children. ${ }^{2,8,9}$ Removal of calculi by SE enables the preservation of the gland as well as its function. Cases of calculi without successful endoscopic treatment are treated with surgical removal of the gland. In this review, the authors were unanimous in suggesting that SE should be the first procedure to be indicated before resection of the gland in children with a diagnosis of SL. ${ }^{2,8,9}$ Variations in the efficacy of SL treatment using SE can be explained by characteristics of each service, including type of equipment, technique used, and staff training, as well as structural abnormalities of the salivary ducts such as stenoses and dilations or size of the calculi.

Regarding the anesthetic technique recommended for the realization of SE in children, we have identified a single author who reports a series in which the procedures were performed under local anesthesia. ${ }^{5}$ This study limited the inclusion of patients with a minimum age of 8 years. Of the nine children included in the study, two did not allow the completion of the procedure because they felt physical discomfort. ${ }^{5}$ Therefore, SE performed under local anesthesia in children can be done under certain controlled conditions, but this must be considered only as an alternative possibility, the rule is indicating the procedure under general anesthesia.

In our opinion, the limitations of this review include the design of the studies, since all seven papers included in our review are case series reports. Thus, their characteristics are not comparable because in case series interventions are not fully symmetrical, there is a certain degree of variation in the criteria for patient allocation and sample characterization, as well as variations regarding experience and technique used in each service.

\section{Conclusion}

Sialendoscopy is a safe and effective procedure for the diagnosis and treatment of recurrent inflammatory diseases of salivary glands in children. 


\section{Resumo}

Endoscopia de glândulas salivares em crianças: revisão sistemática

Objetivo: revisar os estudos sobre endoscopia das glândulas salivares em crianças tendo como foco principal as indicações, os achados endoscópicos e a eficácia do procedimento. Método: foram avaliadas as bases de dados eletrônicas Pubmed, Scielo e Cochrane. A busca foi realizada por dois pesquisadores de forma independente, seguindo critérios de inclusão e exclusão. Um terceiro autor analisou pontos de conflito. Em uma primeira etapa, foram descartados pela leitura do título artigos que não tivessem relação com o objetivo do estudo e a seguir foram avaliados os resumos de cada estudo. Nessas duas fases iniciais, foram excluídos 37 artigos. Os artigos não excluídos pelos critérios de seleção foram levantados e avaliados integralmente. Sete artigos tiveram os dados extraídos e comparados.

Resultados: a busca na literatura de acordo com os parâmetros elencados permitiu a recuperação de 44 artigos. Após a aplicação dos critérios de exclusão, sete estudos foram incluídos na revisão, representando um N de 207 pacientes submetidos à sialoendoscopia (SE) com idades variando de 1 a 16 anos. Todos os estudos, exceto um, realizaram a SE sob anestesia geral. A parotidite recorrente da infância foi a hipótese diagnóstica clínica que levou à indicação de SE no maior número de pacientes, um total de 152 procedimentos. O critério de indicação dos procedimentos foi o número de crises inflamatórias por paciente por ano. A eficácia do procedimento foi consi- derada alta por todos os autores, variando entre 83 e $93 \%$ nas maiores séries avaliadas.

Conclusão: a sialoendoscopia é um procedimento eficaz e seguro para diagnóstico e tratamento de afecções inflamatórias recorrentes de glândulas salivares em crianças.

Palavras-chave: criança, endoscopia/métodos, doenças das glândulas salivares.

\section{References}

1. Jabbour N, Tibesar R, Lander T, Sidman J. Sialendoscopy in children. Int J Pediatr Otorhinolaryngol. 2010, 74(4):347-50.

2. Faure F, Querin S, Dulguerov P, Froehlich P, Disant F, Marchal F. Pediatric salivary gland obstructive swelling: sialendoscopic approach. Laryngoscope. 2007; 117(8):1364-7.

3. Nahlieli O, Baruchin AM. Sialoendoscopy: three years' experience as a diagnostic and treatment modality. J Oral Maxillofac Surg. 1997; 55(9):912-8.

4. Ardeklan L, Klein H, Al Abri R, Marchal F. Sialendoscopy for the diagnosis and treatment of juvenile recurrent parotitis. Rev Stomatol Chir Maxillofac Chir Orale. 2014; 115(1):17-21.

5. Konstantinidis I, Chatziavramidis A, Tsakiropoulou E, Malliari H, Constantinidis J. Pediatric sialendoscopy under local anesthesia: limitations and potentials. Int J Pediatr Otorhinolaryngol. 2011; 75(2):245-9.

6. Shacham R, Droma EB, London D, Bar T, Nahlieli O. Long-term experience with endoscopic diagnosis and treatment of juvenile recurrent parotitis. J Oral Maxillofac Surg. 2009; 67(1):162-7.

7. Capaccio P, Sigismund PE, Luca N, Marchisio P, Pignataro L. Modern management of juvenile recurrent parotitis. J Laryngol Otol. 2012; 126(12):1254-60.

8. Hackett AM, Baranano CF, Reed M, Duvvuri U, Smith RJ, Mehta D Sialoendoscopy for the treatment of pediatric salivary gland disorders. Arch Otolaryngol Head Neck Surg. 2012; 138(10):912-5.

9. Martins-Carvalho C, Plouin-Gaudon I, Quenin S, Lesniak J, Froehlich P, Marchal F, et al. Pediatric sialendoscopy: a 5-year experience at a single institution. Arch Otolaryngol Head Neck Surg. 2010; 136(1):33-6.

10. Bruch JM, Setlur J. Pediatric sialendoscopy. Adv Otorhinolaryngol. 2012; 73:149-52.

11. Becker M, Marchal F, Becker CD, Dulguerov P, Georgakopoulos G, Lehmann W, et al. Sialolithiasis and salivary ductal stenosis: diagnostic accuracy of MR sialography with a three-dimensional extended-phase conjugatesymmetry rapid spin-echo sequence. Radiology. 2000; 217(2):347-58. 